\title{
Singularities on the brane are not isotropic
}

\author{
Marco Bruni \\ Institute of Cosmology and Gravitation, University of Portsmouth, Mercantile House, Portsmouth PO1 2EG, United Kingdom \\ Peter K. S. Dunsby \\ Department of Mathematics and Applied Mathematics, University of Cape Town, Rondebosh 7701, Cape Town, South Africa \\ and South African Astronomical Observatory, Observatory 7925, Cape Town, South Africa
}

(Received 20 July 2002; published 14 November 2002)

\begin{abstract}
Recent studies of homogeneous anisotropic universe models in the brane world scenario show that the cosmological singularity in this context is isotropic. It has therefore been suggested that this may be a generic feature of singularities on the brane, even in the inhomogeneous case. Using a perturbative approach, we show that this is not the case. As in the GR case, the presence of decaying modes in the perturbations signal the instability (in the past) of the isotropic singularity. The brane universe is therefore not born with isotropy built in: as in standard cosmology, the observed large-scale isotropy and homogeneity remains to be explained.
\end{abstract}

DOI: $10.1103 /$ PhysRevD.66.101301

PACS number(s): $98.80 . \mathrm{Cq}$

\section{INTRODUCTION}

The brane world scenario has recently received attention as a possible string inspired cosmology (see [1] for a review). In this scenario the observable universe is a 4-dimensional (4D) slice, the brane, in a higher dimensional spacetime, the bulk. Here we consider the particular implementation developed in [2] in order to generalize a previous model by Randall and Sundrum [3], where the bulk is 5-dimensional (5D) and contains only a cosmological constant, assumed to be negative. In this context various authors [4-9] have considered an homogeneous and anisotropic brane, finding an intriguing result: unlike general relativity, where in general the cosmological singularity is anisotropic, the past attractor for homogeneous anisotropic models in the brane is a simple Robertson-Walker model. In particular, it is found in $[8,9]$ that this is also true for Bianchi type IX models, as well as for some inhomogeneous models.

In general relativity the Belinski-Lifshitz-Kalatnikov conjecture [10] suggests that the Bianchi type IX behavior in the vicinity of the singularity is general, i.e., that the approach to the cosmological singularity in a generic inhomogeneous universe model should locally be the same as in Bianchi type IX.

Building on this and on the fact that Bianchi type IX models in the brane have isotropic singularities, it has been suggested in $[8,9]$ that the isotropic singularity could be a generic feature of brane cosmological models.

A well known problem of cosmology is to explain the very high degree of isotropy observed in the cosmic microwave background (CMB). In a theory such as general relativity, where isotropy is a special rather than generic feature of cosmological models, we need a dynamical mechanism able to produce isotropy. Inflation was proposed, among other reasons, as a way to isotropise the universe. Inflation is effective in this sense, ${ }^{1}$ but it needs homogeneous enough initial data in order to start at all [12]. Although one can adopt the view that one such homogeneous enough patch in an otherwise inhomogenous initial universe is all we need to explain what we observe, this seems somehow unsatisfactory: the isotropy problem remains open in standard cosmology.

If the conjecture in $[8,9]$ could be proved correct, brane cosmology would have the very attractive feature of having isotropy built in. Inflation in this context would still be the most likely way of producing the fluctuations seen in the CMB, but there would be no need of special initial conditions for it to start. ${ }^{2}$ Also, Penrose conjecture [14] on gravitational entropy and an initially vanishing Weyl tensor would be satisfied, cf. [15].

Unfortunately for the brane scenario, we prove here that the past attractor of homogeneous models found in $[8,9]$ is unstable in the past to generic (i.e., anisotropic and inhomogeneous) perturbations. As in general relativity, there is a decaying mode in scalar perturbations that grows unbounded in the past and that signal, in the context of linear perturbation theory, that anisotropy also grows unbounded as $t \rightarrow 0$.

In order to prove this, we specifically look at perturbation modes of the dimensionless shear $\sigma / H$, and show that there is a decaying mode (growing in the past) in this quantity. Ours is a large-scale analysis, at a time when physical scales of perturbations are much larger than the Hubble radius, $\lambda$ $\gg H^{-1}$ (equivalent to neglecting Laplacian terms in the evolution equations). This may seem restrictive, but this is not the case for the noninflationary perfect fluid models that are relevant to our discussion. Indeed in this case any wave-

\footnotetext{
${ }^{1}$ There are perturbative proofs of the cosmic no-hair conjecture, i.e., classical perturbations in inflationary models with a scalar field or cosmological constant are swept out, as well as (local) proofs for homogeneous and inhomogeneous models (see e.g. [11] and references therein).

${ }^{2}$ See [13] for CMB limits on the anisotropy in brane cosmology.
} 
length $\lambda$, smaller than $H^{-1}$ at a given time, becomes much larger than $H^{-1}$ at earlier enough times. Because of this crucial property of perturbations for noninflationary models our analysis is completely general, i.e., valid for any $\lambda$ as $t$ $\rightarrow 0$.

For the most part we follow the notation and convention of $[1,16,17]$. In Sec. II we briefly summarize those results on general brane dynamics that are relevant for the following discussion (see $[1,2,16]$ for more details and other references). In Sec. III we present the equations for scalar perturbations and derive the large-scale evolution for the gaugeinvariant density perturbation variable in the high energy limit. In particular we highlight the decaying mode that grows unbounded in the past. In Sec. IV we show that a corresponding mode in the dimensionless shear $\sigma / H$ also grows in the past. In Sec. V we draw our conclusions.

\section{BRANE DYNAMICS}

The implementation of the brane-world scenario considered in [2] assumes that the whole spacetime is 5D and governed by 5D Einstein field equations:

$$
\widetilde{G}_{A B}=\widetilde{\kappa}^{2}\left[-\tilde{\Lambda} \tilde{g}_{A B}+\delta_{A B}(\chi)\left(-\lambda g_{A B}+T_{A B}\right)\right] .
$$

These represent a 4D brane at $\chi=0$ living in a bulk with metric $\tilde{g}_{A B}$ and cosmological constant $\tilde{\Lambda} ; \widetilde{\kappa}^{2}$ is the $5 \mathrm{D}$ gravitational constant, $\lambda$ is the brane tension, $g_{A B}$ and $T_{A B}$ are respectively the metric and the energy-momentum on the brane. The 4D field equations induced on the brane are derived geometrically from Eq. (1) assuming a $Z_{2}$ symmetry with the brane as the fixed point, leading to modified Einstein equations with new terms representing bulk effects:

$$
G_{\mu \nu}=-\Lambda g_{\mu \nu}+\kappa^{2} T_{\mu \nu}+\tilde{\kappa}^{4} S_{\mu \nu}-\mathcal{E}_{\mu \nu},
$$

where as usual $\kappa^{2}=8 \pi / M_{\mathrm{p}}^{2}$. The various physical constants and parameters appearing in the equations above are not independent, but related to each other by

$$
\lambda=6 \kappa^{2} / \widetilde{\kappa}^{4}, \Lambda=\frac{1}{2} \tilde{\kappa}^{2}\left(\widetilde{\Lambda}+\frac{1}{6} \tilde{\kappa}^{2} \lambda^{2}\right) .
$$

The tensor $S_{\mu \nu}$ represents nonlinear matter corrections given by

$$
S_{\mu \nu}=\frac{1}{12} T_{\alpha}{ }^{\alpha} T_{\mu \nu}-\frac{1}{4} T_{\mu \alpha} T^{\alpha}{ }_{\nu}+\frac{1}{24} g_{\mu \nu}\left[3 T_{\alpha \beta} T^{\alpha \beta}-\left(T_{\alpha}{ }^{\alpha}\right)^{2}\right] .
$$

$\mathcal{E}_{\mu \nu}$ is the projection on the brane of the 5D Weyl tensor; although the whole dynamics is $5 \mathrm{D}$ and given by Eq. (1), from the $4 \mathrm{D}$ point of view $\mathcal{E}_{\mu \nu}$ is a nonlocal source term that carries bulk effects onto the brane. If $u^{\mu}$ is the 4-velocity of matter and $h_{\mu \nu}=g_{\mu \nu}+u_{\mu} u_{\nu}$ projects into the comoving rest space, one can decompose $\mathcal{E}_{\mu \nu}$ as $[1,16]$

$$
\mathcal{E}_{\mu \nu}=\frac{-6}{\kappa^{2} \lambda}\left[\mathcal{U}\left(u_{\mu} u_{\nu}+\frac{1}{3} h_{\mu \nu}\right)+\mathcal{P}_{\mu \nu}+\mathcal{Q}_{\mu} u_{\nu}+\mathcal{Q}_{\nu} u_{\mu}\right],
$$

effectively as if it was a traceless energy momentum tensor with energy density $\mathcal{U}$, energy flux $\mathcal{Q}_{\mu}$ and anisotropic pressure $\mathcal{P}_{\mu \nu}$. The brane energy-momentum tensor separately satisfies the conservation equations, $\nabla^{\nu} T_{\mu \nu}=0$. Assuming a perfect fluid (or minimally coupled scalar field) we have the usual results

$$
\begin{array}{r}
\dot{\rho}+\Theta(\rho+p)=0, \\
\mathrm{D}_{\mu} p+(\rho+p) A_{\mu}=0,
\end{array}
$$

where a dot denotes $u^{\nu} \nabla_{\nu}, \Theta=\mathrm{D}^{\mu} u_{\mu}$ is the volume expansion, $A_{\mu}=\dot{u}_{\mu}$ is the 4-acceleration, and $\mathrm{D}_{\mu}$ denotes the spatially projected covariant derivative. The contracted Bianchi identities on the brane then imply that the projected Weyl tensor $\mathcal{E}_{\mu \nu}$ and $S_{\mu \nu}$ obey the constraints

$$
\nabla^{\mu} \mathcal{E}_{\mu \nu}=\frac{6 \kappa^{2}}{\lambda} \nabla^{\mu} S_{\mu \nu}
$$

which show how the nonlocal bulk effects are sourced by the evolution and spatial inhomogeneity of the brane matter content. Finally, using Eqs. (2)-(7), these can be turned in propagation equations for the nonlocal energy density $\mathcal{U}$ and energy flux $\mathcal{Q}_{\mu}$. Neglecting terms quadratic in covariant variables that are gauge-invariant perturbations with respect to a Robertson-Walker isotropic background $[16,18,19]$, these are $^{3}$

$$
\dot{\mathcal{U}}+\frac{4}{3} \Theta \mathcal{U}+\mathrm{D}^{\mu} \mathcal{Q}_{\mu}=0,
$$

$\dot{\mathcal{Q}}_{\mu}+4 H \mathcal{Q}_{\mu}+\frac{1}{3} \mathrm{D}_{\mu} \mathcal{U}+\frac{4}{3} \mathcal{U} A_{\mu}+\mathrm{D}^{\nu} \mathcal{P}_{\mu \nu}=-\frac{1}{6} \kappa^{4}(\rho+p) \mathrm{D}_{\mu} \rho$

where $H=\dot{a} / a\left(=\frac{1}{3} \Theta\right)$ is the Hubble expansion of the background. Using a standard decomposition of perturbations, the anisotropic term $\mathcal{P}_{\mu \nu}$ carries in general contributions from scalar, vector and tensor modes; the latter however, satisfying $\mathrm{D}^{\nu} \mathcal{P}_{\mu \nu}=0$, does not contribute the nonlocal conservation equations above.

In the background, the Raychaudhuri equation is

$\dot{H}=-H^{2}-\frac{\kappa^{2}}{6}\left[\rho+3 p+\frac{\rho}{\lambda}(2 \rho+3 p)\right]+\frac{1}{3} \Lambda-\frac{2}{\kappa^{2} \lambda} \mathcal{U}_{o}\left(\frac{a_{o}}{a}\right)^{4}$,

where the solution for $\mathcal{U}$ follows from Eq. (9) (considering the zero order background only), $a_{o}$ is the initial scale factor and $\mathcal{U}_{o}=\mathcal{U}\left(a_{o}\right)$. The first integral of this equation is the generalized Friedmann equation on the brane $(K=0, \pm 1)$ :

\footnotetext{
${ }^{3}$ Strictly speaking, the variables defined in [18-20] and those defined in the same way in the brane context $[1,16,21]$ are $4 \mathrm{D}$; they are however easily generalized to 5D. Bardeen-like variables $[22,23]$ have been defined in 5D in order to carry out a brane-bulk analysis, e.g., see [24], but the relation between these and the covariant ones used here has not yet been established; cf. $[19,20]$ for this relation in $4 \mathrm{D}$ general relativity.
} 


$$
H^{2}=\frac{\kappa^{2}}{3} \rho\left(1+\frac{\rho}{2 \lambda}\right)+\frac{1}{3} \Lambda-\frac{K}{a^{2}}+\frac{2}{\kappa^{2} \lambda} \mathcal{U}_{o}\left(\frac{a_{o}}{a}\right)^{4}
$$

The high energy regime is defined by $\rho \gg \lambda$. In this limit one obtains flat models dominated by the non-linear $\rho$ term [25],

$$
H^{2}=\left(\kappa^{2} / 6 \lambda\right) \rho^{2}, \quad a=\left(t / t_{o}\right)^{1 / 3(1+w)},
$$

where we fix an arbitrary initial condition by choosing $a_{o}$ $=a\left(t_{o}\right)=1$, and as usual $w=p / \rho$. These models are represented by a stationary (equilibrum) point, denoted $\mathcal{F}_{b}$, in the phase space of homogeneous Bianchi models considered in $[8,9]$, as well as in the phase space of a special class of inhomogeneous $G_{2}$ cosmological models. In both cases $\mathcal{F}_{b}$ is found to be the source, or past attractor, for the generic dynamics for $w>0$ ( $w=0$ is also included in the homogeneous case), consistently with [4-7].

Finally, we note that the condition for inflation in general is [26]

$$
w<-\frac{1}{3}\left(\frac{2 \rho+\lambda}{\rho+\lambda}\right) .
$$

As $\rho / \lambda \rightarrow \infty$ this becomes $w<-\frac{2}{3}$, while the general relativity condition $w<-\frac{1}{3}$ is recovered as $\rho / \lambda \rightarrow 0$.

\section{DENSITY PERTURBATIONS}

Scalar gauge-invariant perturbations can be described using covariantly defined variables (see $[18,19]$ and references therein). In the brane scenario this formalism has been developed in $[1,16]$ (see also [21]); here we shall follow the same approach, with minor modifications, and we refer to these papers for definitions.

We can completely characterize scalar perturbations on the brane with four variables, $\Delta, C, U$ and $Q$, representing respectively the matter density perturbation, a convenient 3-curvature perturbation, the perturbation of the Weyl energy density, and a Weyl energy flux variable related to $\mathcal{Q}_{\mu}$ in Eq. (5) (all 3-quantities are defined with respect to a single 4-velocity field, that of matter $u^{\mu}$ ). The dynamics of these quantities is given by

$$
\begin{aligned}
\dot{\Delta}= & {\left[3 w H-\frac{\kappa^{2} \rho(1+w)}{2 H}\left(1+\frac{\rho}{\lambda}\right)\right] \Delta+\frac{(1+w)}{4 a^{2} H} C } \\
& -\frac{\rho(1+w)}{2 H}\left(\frac{6}{\lambda \kappa^{2}}\right) U \\
\dot{C}= & \left(\frac{4 a^{2} H c_{\mathrm{s}}^{2}}{1+w}\right) \mathrm{D}^{2} \Delta-\left(\frac{12 a^{3} \rho}{\lambda \kappa^{2}}\right) \mathrm{D}^{2} Q \\
& -\left(\frac{72 a^{2} H c_{\mathrm{s}}^{2}}{1+w}\right)\left(\frac{\mathcal{U}}{\lambda \kappa^{2}}\right) \Delta,
\end{aligned}
$$

$$
\begin{aligned}
\dot{U}= & (3 w-1) H U-\left(\frac{4 c_{\mathrm{s}}^{2}}{1+w}\right)\left(\frac{\mathcal{U}}{\rho}\right) H \Delta \\
& -\left(\frac{4 \mathcal{U}}{3 \rho}\right) Z-a \mathrm{D}^{2} Q \\
\dot{Q}= & (1-3 w) H Q-\frac{1}{3 a} U-\frac{2}{3} a \mathrm{D}^{2} P \\
& +\frac{1}{6 a}\left[\left(\frac{8 c_{\mathrm{s}}^{2}}{1+w}\right) \frac{\mathcal{U}}{\rho}-\kappa^{4} \rho(1+w)\right] \Delta .
\end{aligned}
$$

Note that this system of equation is homogeneous in the four chosen variables, except for the $P$ term in Eq. (18); this represents the contribution from the anisotropic Weyl stress, and since there is no evolution equation for $P$, in general one should either determine $P$ from the full 5D dynamics, or make some ansatz; otherwise, in general the system above is not closed and one cannot find solutions. Finally, the variable $Z$ in Eq. (17), used in $[1,16,17]$ and characterizing the perturbation of the expansion, is related by $\Delta, U$ and $C$ by the constraint

$$
C=2 \kappa^{2} a^{2} \rho(1+\rho / \lambda) \Delta+\left(12 / \lambda \kappa^{2}\right) a^{2} \rho U-4 a^{2} H Z,
$$

arising from the Gauss-Codazzi constraint in the brane.

In the following we want to study the stability properties of the models $\mathcal{F}_{b}$, Eq. (13), against generic inhomogeneous and anisotropic perturbations, for values of $w \geqslant 0$. We see from Eq. (14) that these models are non-inflationary for $w$ $\geqslant 0$. Thus, as we said in the Introduction, for any $\lambda$ we only need to study the large-scale evolution of the variables above. We can either neglect the Laplacian terms in Eqs. (15)-(18), or use a harmonic expansion (Fourier in our flat space case) and neglect terms $H^{-2} / \lambda^{2} \ll 1$ : the resulting equations are the same.

Fortunately, in the large-scale limit the $\mathrm{D}^{2} Q$ term in Eq. (17) is negligible, and one obtains a closed system for the density perturbations $\Delta$ and $U$ and the curvature variable $C$. Besides, to the extent that the $P$ contribution to Eq. (18) is also negligible in this limit, cf. [27], $Q$ can also be determined $[1,16,17]$. In addition, we now restrict our analysis to the case $\mathcal{U}=0$ : we will comment on the reliability of this assumption in the conclusions.

Introducing the new variables $\widetilde{U}=U /\left(\kappa^{4} \rho\right)$ and $\widetilde{Q}$ $=a H Q / \kappa^{4} \rho$ and denoting with a prime the derivative with respect to $d \tau=d \ln (a)$, we have

$$
\begin{aligned}
& \Delta^{\prime}= {\left[3 w-\frac{\kappa^{2} \rho(1+w)}{2 H^{2}}\left(1+\frac{\rho}{\lambda}\right)\right] \Delta } \\
&+\frac{(1+w)}{4 a^{2} H^{2}} C-\frac{3(1+w) \kappa^{2} \rho^{2}}{\lambda H^{2}} \widetilde{U}, \\
& C^{\prime}= 0, \\
& \widetilde{U}^{\prime}=2(3 w+1) \widetilde{U},
\end{aligned}
$$




$$
\widetilde{Q}^{\prime}=(2-3 w) \widetilde{Q}-\frac{1}{3} \widetilde{U}-\frac{1}{6}(1+w) \Delta
$$

We see therefore that the evolution in the long wavelength limit, with $\mathcal{U}=0$, considerably simplifies: it is described by a single first order equation for the density perturbation and two first integrals arising from Eqs. (21),(22). The first, $C_{\mathrm{o}}$, represent the large-scale constant spatial curvature perturbation; the second, $\widetilde{U}_{\mathrm{o}}=\widetilde{U} a^{-2(1+3 w)}$, is a first integral for the Weyl energy density perturbation. These source the density perturbation; therefore, $\Delta$ has three modes, as opposed to the two arising in GR in the case of a single fluid. The first two are analogous to the GR ones: the first is the mode arising from the $C_{\mathrm{o}}=\widetilde{U}_{\mathrm{o}}=0$ initial condition, typically a "decaying mode," the second is the curvature adiabatic mode generated by $C_{0}$; the third mode is a peculiarity of the brane scenario, is generated by $\widetilde{U}_{0}$, and represents an isocurvature (or entropy) perturbation. Although this is not particularly clear from the treatment used here and in $[1,16,17]$, this mode is indeed due to the different intrinsic 4-velocities that the "Weyl fluid" and matter have in the perturbed spacetime (which is the cause of the presence of the energy flux variable $Q$ ). In complete analogy with the case of two fluids in GR, this then generates a "relative entropy perturbation" (see e.g. [20,23] and [17]). Finally, and again as in GR, we remark that the adiabatic and isocurvature perturbations evolve independently as described above only in the long wavelength limit: it is indeed clear from Eqs. (15)-(18) that in general the two modes are coupled and nonvanishing even starting from $C_{\mathrm{o}}=\widetilde{U}_{\mathrm{o}}=0$. Conversely the decaying mode should be seen as arising from special initial conditions that lead to vanishing values of $C$ and $\widetilde{U}$ when $\lambda \gg H^{-1}$. Usually this mode is decaying forward in time, and therefore neglected in structure formation studies, while the growing mode is the interesting one. In studying the question of homogeneity and isotropy of the brane at early times we are interested in running the equations above backwords in time, and it is the decaying mode that plays a crucial role for most values of $w$, as we are now going to show.

Let us now restrict our analysis to the high-energy regime that dominates at very early times, when the background is given by the models $\mathcal{F}_{b}$, Eq. (13). Using $C_{\mathrm{o}}$ and $\widetilde{U}_{\mathrm{o}}$, in the limit $\rho \gg \lambda$ the evolution of $\Delta$ is determined by the simple equation

$$
\Delta^{\prime}=-3 \Delta+\frac{9}{4}(1+w)^{3} C_{\mathrm{o}} a^{q}-18(1+w) \widetilde{U}_{\mathrm{o}} a^{r},
$$

from which the three density perturbation modes are immediately determined. They are

$$
\Delta=\Delta_{\mathrm{o}} a^{p}+\frac{9}{4} \frac{(1+w)^{3}}{6 w+7} C_{\mathrm{o}} a^{q}-\frac{18(1+w)}{6 w+5} \widetilde{U}_{\mathrm{o}} a^{r},
$$

where

$$
\begin{gathered}
p=-3, \quad q=6 w+4, \quad r=2(1+3 w), \\
q<0 \Leftrightarrow w<-\frac{2}{3}, \quad r<0 \Leftrightarrow w<-\frac{1}{3},
\end{gathered}
$$

and $\Delta_{\mathrm{o}}$ is the constant of integration associated with the decaying mode. This shows that independently of the value of $w$, there is always a large-scale mode that grows unbounded in the past.

The solution for $\widetilde{Q}$ can also be easily determined:

$$
\begin{aligned}
\widetilde{Q}= & \widetilde{Q}_{\mathrm{o}} a^{s}+\frac{1}{6} \frac{1+w}{5-3 w} \Delta_{\mathrm{o}} a^{p}-\frac{3}{8} \frac{(1+w)^{4}}{(7+6 w)(2+9 w)} C_{\mathrm{o}} a^{q} \\
& +\frac{1}{27} \frac{(2+3 w)^{2}}{w(5+6 w)} \widetilde{U}_{\mathrm{o}} a^{r}
\end{aligned}
$$

for $w \neq 0$ and

$$
\widetilde{Q}=\widetilde{Q}_{\mathrm{o}} a^{2}+\frac{1}{30} \Delta_{\mathrm{o}} a^{-3}-\frac{3}{112} C_{\mathrm{o}} a^{4}+\frac{4}{45} \widetilde{U}_{\mathrm{o}} a^{2} \ln a
$$

for $w=0$, where

$$
s=2-3 w, \quad s<0 \Leftrightarrow w>\frac{2}{3},
$$

and $\widetilde{Q}_{\mathrm{o}}$ is a constant of integration representing the homogeneous solution to Eq. (23).

\section{THE EXPANSION NORMALIZED SHEAR}

Like in the standard GR case, all the gauge-invariant geometric and kinematic quantities can be expressed in terms of $\Delta[28]$ in the context of linear perturbation theory. The key covariant variable related to the issue of isotropization in the past is the expansion normalized shear [29],

$$
\Sigma_{a b}=\sigma_{a b} / H .
$$

The scalar contribution to this quantity is obtained by taking its total spatial divergence [19]:

$$
\Sigma=a^{2} D^{a} D^{b} \sigma_{a b} / H
$$

Using the shear constraint equation [Eq. (97) in Ref. [1]] it is easy to show that $\Sigma$ can be expressed in terms of $\Delta, C, \widetilde{U}$ and $\widetilde{Q}$ :

$$
\Sigma=2 \Delta-\left(1 / 4 a^{2} H^{2}\right) C+12 \widetilde{U}+36 \widetilde{Q},
$$

and using Eqs. (25) and (28) together with the solutions for $\widetilde{U}$ and $\widetilde{Q}$ we obtain

$$
\begin{aligned}
\Sigma= & \frac{16}{5-3 w} \Delta_{\mathrm{o}} a^{p}-\frac{3}{4} \frac{(1+w)^{2}\left(99 w^{2}+153 w+40\right)}{(6 w+7)(2+9 w)} C_{\mathrm{o}} a^{q} \\
& +\frac{8}{3} \frac{18 w^{2}+15 w+2}{w(62+5)} \widetilde{U}_{\mathrm{o}} a^{r}+36 \widetilde{Q}_{\mathrm{o}} a^{s}
\end{aligned}
$$

for $w \neq 0$ and

$$
\Sigma=\frac{16}{5} \Delta_{\mathrm{o}} a^{-3}-\frac{51}{28} C_{\mathrm{o}} a^{4}+\frac{24}{5} \widetilde{U}_{\mathrm{o}}(1+2 \ln a) a^{2}+36 \widetilde{Q}_{\mathrm{o}} a^{2}
$$

for $w=0$. The presence of the decaying mode $p=-3$ in Eqs. (34) and (35) proves that $\Sigma$ grows in the past. This 
completes our proof that the past attractor $\mathcal{F}_{b}$ of homogeneous models is unstable in the past against anisotropic and inhomogeneous perturbations.

\section{CONCLUSIONS}

From a dynamical system point of view the past attractor $\mathcal{F}_{b}$ for brane homogeneous cosmological models found in $[8,9]$ is a fixed point in the phase space of these models. This phase space may be thought of as an invariant submanifold within a higher dimensional phase space for more general inhomogeneous models. The conjecture in $[8,9]$ is equivalent to saying, in this dynamical system language, that $\mathcal{F}_{b}$ is the (local) past attractor for generic trajectories in this higher dimensional phase space. Our analyisis can be seen as an exploration of the neighborhood of $\mathcal{F}_{b}$ out of the invariant submanifold explored in $[8,9]$. We have found that $\mathcal{F}_{b}$ is unstable in the past to generic anisotropic and inhomogeneous perturbations of noninflationary perfect fluid models with $p=w \rho$, for any value of $w$, using a large-scale $\lambda$ $\gg H^{-1}$ approximation that we have motivated in the Introduction and is not restricting the validity of our analysis. The instability of $\mathcal{F}_{b}$ we have found is fundamentally due, among other modes that may be stable or not depending on the value of $w$, to a decaying mode in the density perturbation that blows up in the past, $\Delta \sim a^{-3}$, in a way independent of $w$ and that, like in general relativity, is the signal of the unbounded growth of the dimensionless shear $\sigma / H$ as $t \rightarrow 0$, as proved in Sec. IV.
We have considered here only the case of vanishing background Weyl energy density, $\mathcal{U}=0$. This assumption considerably simplifies the analysis, but it is an easy guess that our results will remain true for $\mathcal{U} \neq 0$. Indeed when $\mathcal{U} \neq 0, \mathcal{F}_{b}$ still remains the past attractor of the isotropic models whose stability in the past we want to examine, as is clear from the Friedmann equation (12). In other words, our analysis is restricted to the invariant submanifold $\mathcal{U}=0$ of the larger phase space with $\mathcal{U} \neq 0$, but this submanifold is asymptotically stable against $\mathcal{U} \neq 0$ perturbations.

Finally, it has recently been suggested [30] that the quantity $\mathcal{U}_{0}$ in Eq. (12) is only asymptotically a constant, while $\mathcal{U}_{0} \sim a^{4}$ at high enough energies. As is clear from Eq. (12), even more in this case $\mathcal{F}_{b}$ still remains the relevant past attractor of isotropic models. We believe therefore that our analyisis should remain valid also in this case. A more complete analysis including this issue and therefore that of $\mathcal{U}$ $\neq 0$ will be the subject of a future investigation.

\section{ACKNOWLEDGMENTS}

M.B. thanks Roy Maartens, Carlos Sopuerta, Filippo Vernizzi, and David Wands for useful discussions, and the Department of Mathematics and Applied Mathematics of the University of Cape Town and the Albert Einstein Institute (Golm) for hospitality while part of this work was carried out. P.K.S.D. thanks NRF (South Africa) for financial support.
[1] R. Maartens, in Reference Frames \& Gravitomagnetism, edited by J. Pascual-Sanchez et al. (World Scientific, Singapore, 2001), p. 93, gr-qc/0101059.

[2] T. Shiromizu, K.I. Maeda, and M. Sasaki, Phys. Rev. D 62, 024012 (2000).

[3] L. Randall and R. Sundrum, Phys. Rev. Lett. 83, 4690 (1999).

[4] R. Maartens, V. Sahni, and T.D. Saini, Phys. Rev. D 63, 063509 (2001).

[5] A. Campos and C.F. Sopuerta, Phys. Rev. D 63, 104012 (2001).

[6] M.G. Santos, F. Vernizzi, and P.G. Ferreira, Phys. Rev. D 64, 063506 (2001).

[7] A. Campos and C.F. Sopuerta, Phys. Rev. D 64, 104011 (2001).

[8] A.A. Coley, Class. Quantum Grav. 19, L45 (2002).

[9] A.A. Coley, Phys. Rev. D 66, 023512 (2002).

[10] V.A. Belinski, I.M. Khalatnikov, and E.M. Lifshitz, Adv. Phys. 19, 525 (1970).

[11] M. Bruni, F.C. Mena, and R. Tavakol, Class. Quantum Grav. 19, L23 (2002).

[12] E.W. Kolb and M.S. Turner, The Early Universe (AddisonWesley, Redwood City, CA, 1990).

[13] J.D. Barrow and R. Maartens, Phys. Lett. B 532, 153 (2002).

[14] R. Penrose, in General Relativity: An Einstein Centenary Survey, edited by S.W. Hawking and W. Israel (Cambridge Uni- versity Press, Cambridge, England, 1979), p. 581.

[15] P. Tod, Class. Quantum Grav. 7, L13 (1990).

[16] R. Maartens, Phys. Rev. D 62, 084023 (2000).

[17] C. Gordon and R. Maartens, Phys. Rev. D 63, 044022 (2001).

[18] G.F.R. Ellis and M. Bruni, Phys. Rev. D 40, 1804 (1989).

[19] M. Bruni, P.K.S. Dunsby, and G.F.R. Ellis, Astrophys. J. 395, 34 (1992).

[20] P.K.S. Dunsby, M. Bruni, and G.F.R. Ellis, Astrophys. J. 395, 54 (1992).

[21] B. Leong et al., Phys. Rev. D 65, 104012 (2002).

[22] J.M. Bardeen, Phys. Rev. D 22, 1882 (1980).

[23] H. Kodama and M. Sasaki, Prog. Theor. Phys. 78, 1 (1984).

[24] H.A. Bridgman, K.A. Malik, and D. Wands, Phys. Rev. D 65 , 043502 (2002).

[25] P. Binétruy, C. Deffayet, and D. Langlois, Nucl. Phys. B565, 269 (2000).

[26] R. Maartens, D. Wands, B.A. Bassett, and I.P.C. Heard, Phys. Rev. D 62, 041301 (2000).

[27] D. Langlois, R. Maartens, M. Sasaki, and D. Wands, Phys. Rev. D 63, 084009 (2001).

[28] S.W. Goode, Phys. Rev. D 39, 2882 (1989).

[29] S.W. Goode and J. Wainwright, Class. Quantum Grav. 2, 99 (1985).

[30] D. Langlois, L. Sorbo, and M. Rodríguez-Martínez, Phys. Rev. Lett. 89, 171301 (2002). 